\title{
Reviewers in this Issue
}

Prof. Iftikhar Ahmed

Department of Microbiology

Enam Medical College, Savar, Dhaka

Prof. Md. Aminul Haque Khan

Department of Biochemistry Enam Medical College, Savar, Dhaka

Col. Mimi Parvin

Classified Specialist

Department of Pathology

Combined Military Hospital

Shaheed Salahuddin Cantonment, Ghatail, Tangail

Prof. Md. Rezwanur Rahman

Department of Biochemistry

Delta Medical College, Dhaka

Prof. Irin Perveen

Department of Gastroenterology

Enam Medical College \& Hospital, Savar, Dhaka

Dr. Md. Atiqur Rahman

Associate Professor

Department of Pathology

Enam Medical College, Savar, Dhaka

Dr. Mithun Alamgir

Associate Professor

Department of Community Medicine

Enam Medical College, Savar, Dhaka

Dr. Rukhsana Parvin

Associate Professor, Department of Medicine Enam Medical College \& Hospital, Savar, Dhaka

Dr. Ishrat Jahan

Associate Professor

Department of Paediatrics

Enam Medical College \& Hospital, Savar, Dhaka 